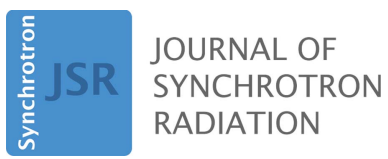

ISSN 1600-5775

Received 22 July 2019

Accepted 7 November 2019

Edited by I. Schlichting, Max Planck Institute for Medical Research, Germany

Keywords: SOLEIL; microfluidics; X-ray microfluorescence; X-ray absorption; smallangle $\mathrm{X}$-ray scattering; macromolecular crystallography.

Supporting information: this article has supporting information at journals.iucr.org/s

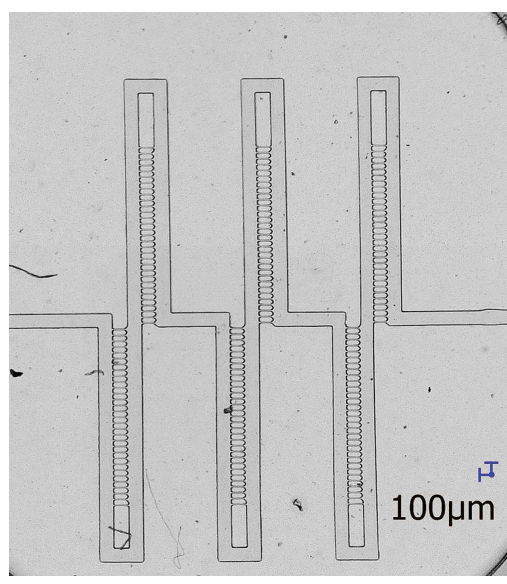

C 2020 International Union of Crystallography

\section{The microfluidic laboratory at Synchrotron SOLEIL}

\author{
Igor Chaussavoine, ${ }^{a}$ Anthony Beauvois, ${ }^{\mathrm{a}, \mathrm{b}, \mathrm{c}}$ Tiphaine Mateo, ${ }^{\mathrm{a}}$ \\ Ramakrishna Vasireddi, ${ }^{a}$ Nadine Douri, ${ }^{a}$ Jordan Priam, ${ }^{a}$ Youssef Liatimi, \\ Stéphane Lefrançois, ${ }^{\mathrm{a}}$ Hervé Tabuteau, ${ }^{\mathrm{d}}$ Mélanie Davranche, \\ Delphine Vantelon, ${ }^{a}$ Thomas Bizien, ${ }^{\mathrm{a} *}$ Leonard, M. G. Chavas ${ }^{\mathrm{a} *}$ and \\ Benedikt Lassalle-Kaiser ${ }^{\mathrm{a} *}$
}

\footnotetext{
${ }^{a}$ Synchrotron SOLEIL, Saint-Aubin, 91192 Gif-sur-Yvette, France, ${ }^{\mathbf{b}}$ Univ. Rennes, CNRS, Géosciences Rennes - UMR 6118, F-35000 Rennes, France, 'Laboratoire Léon Brillouin, Commissariat à l'Energie Atomique Saclay, 91191 Gif-sur-Yvette, France, and dUniv. Rennes, CNRS, IPR (Institut de Physique de Rennes) - UMR 6251, F-35000 Rennes, France. *Correspondence e-mail: delphine.vantelon@synchrotron-soleil.fr,

thomas.bizien@synchrotron-soleil.fr, leonard.chavas@synchrotron-soleil.fr, benedikt.lassalle@synchrotron-soleil.fr
}

A microfluidic laboratory recently opened at Synchrotron SOLEIL, dedicated to in-house research and external users. Its purpose is to provide the equipment and expertise that allow the development of microfluidic systems adapted to the beamlines of SOLEIL as well as other light sources. Such systems can be used to continuously deliver a liquid sample under a photon beam, keep a solid sample in a liquid environment or provide a means to track a chemical reaction in a time-resolved manner. The laboratory provides all the amenities required for the design and preparation of soft-lithography microfluidic chips compatible with synchrotron-based experiments. Three examples of microfluidic systems that were used on SOLEIL beamlines are presented, which allow the use of $\mathrm{X}$-ray techniques to study physical, chemical or biological phenomena.

\section{Introduction}

Microfluidics is a rapidly evolving field that covers a wide range of scientific domains for both applied and fundamental research (Whitesides, 2006). Commercial applications using microfluidics range from biomedical analyses to drug discovery and printing systems, with a particularly high impact on health technologies. Fundamental research also takes advantage of microfluidic systems to explore physical phenomena that can only be accessed under specific physical conditions: interfacial physics (Hemmerle et al., 2016), emulsions (Zarzar et al., 2015) and droplets (Joanicot \& Ajdari, 2005) formation or (bio)chemical kinetics (Song \& Ismagilov, 2003). Microfluidic systems also offer exciting opportunities for spectroscopic, structural and imaging techniques. On the one hand, the possibility to study samples in liquid environments; on the other hand, an access to novel physical properties that are specific to the micrometre scale (Tabeling, 2005). The advances in polymer-based soft-lithography techniques (Qin et al., 2010) now allow designing, producing and analyzing microfluidic systems within less than a day. This short-time production is ideal to meet the time constraints of typical beam time allocation schemes at synchrotrons or freeelectron lasers. These manufacturing procedures offer the possibility to produce disposable chips that meet the requirements of both the system to be studied and the photonbased tool to be used. Such experiments have already been conducted at synchrotrons, where a wide range of photon energies are available with a high brilliance and thus allow 
unique experiments (Zheng et al., 2004; Polte et al., 2010; Reich et al., 2005). However, coupling microfluidic devices with synchrotron techniques is a significant challenge since each photon-based technique has specific requirements such as photon penetration depth, low pressures, sample dimensions or physical constraints. In order to satisfy this increasing need at synchrotron facilities, a microfluidic laboratory was recently opened at SOLEIL. Its aim is to sustainably provide the environment and know-how for the implementation of microfluidic systems in photon-based experiments. It is worth mentioning that the current or upcoming upgrade of several synchrotrons into diffraction-limited storage rings will increase the photon density at the sample, which will require fast sample replacement to either avoid radiation damage or provide a new sample to optimize data-collection strategies.

The present article describes the environment and tools that are available to produce and analyze microfluidic systems in this laboratory. Examples of the first projects carried out using this facility highlight specific research applications where microfluidic devices can be adapted to synchrotron-based techniques to generate new and original approaches. As a prospectus, this article outlines the research areas where we expect microfluidic and synchrotron research to cooperate in the near future.

\section{Facilities and instruments}

The microfluidics laboratory at SOLEIL has a $25 \mathrm{~m}^{2}$ clean room with a controlled environment located in the experimental hall within the synchrotron building (Fig. 1). Separated from the synchrotron hall by a double-door entry, with the air temperature and quality controlled, the laboratory's ceiling illuminations and windows are filtered to absorb more than $90 \%$ of the natural UV light. The room is equipped with all the required instrumentation for basic chemical or biochemical work (fume hood, laminar flow cabinet, water purifying system, solvent cabinet, waste containers, refrigerator, oven and hot plates). The clean room was classified as class 10.000/ ISO 7 (US FED/ISO system).

The purpose of the microfluidic laboratory is to host and maintain instruments for the fabrication and analysis of

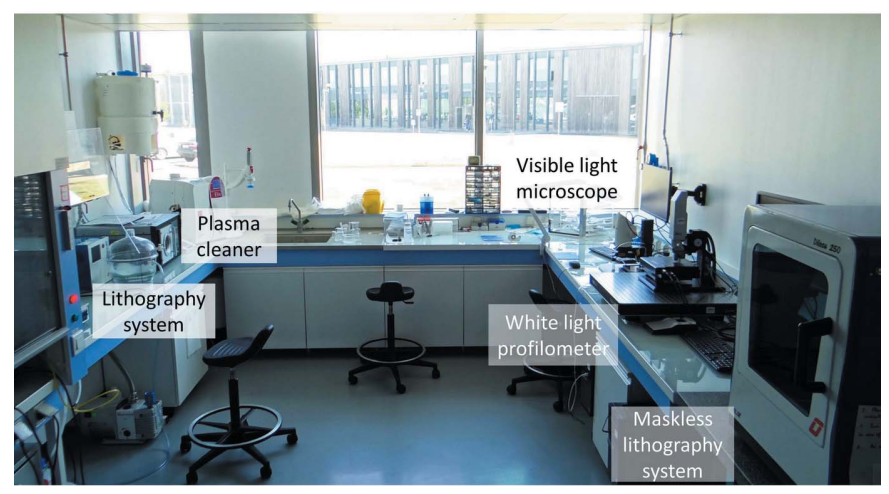

Figure 1

Overview of the clean room of the microfluidic laboratory. microfluidic systems applied to synchrotron-based experiments. In this prospect, micro-fabrication processes can be performed using the following apparatus.

(i) A spin coater (Laurell Technologies) with a maximum rotation speed of 12000 r.p.m., which allows producing thin films (down to tens of nanometres) on substrates (e.g. silicon wafers or glass plates).

(ii) A direct maskless lithography system (Dilase-250, KLOE), which allows the patterning of photosensitive resins. The laser wavelength is $375 \mathrm{~nm}$ for a maximum power of $18 \mathrm{~mJ}$. The laser spot size can be tuned from 4 to $50 \mu \mathrm{m}$. The time required for the patterning of a complete microfluidic system using this device is much higher than using a traditional light-exposure device using masks (vide infra) since the laser system literally has to draw the whole pattern including filling large areas. The choice of this instrument was motivated by its flexibility in fabricating patterns in a minimal amount of time directly from the design step to the printed pattern, as opposed to delays in purchasing a mask from an external company.

(iii) A hydraulic high-temperature press (Vogt $\mathrm{GmbH}$ ), which allows producing microfluidic chips based on COC (cyclic olefins copolymers). Using COC is particularly interesting for chips that are destined for synchrotron experiments since the thickness of the chip window can be as thin as $10 \mu \mathrm{m}$, thus minimizing the background.

(iv) A UV-light-exposure system (UV-KUB 2, KLOE) is available for photolithography processes using dedicated masks.

(v) A plasma-generating device (Henniker Ltd) that allows $\mathrm{O}_{2}, \mathrm{~N}_{2}$, Ar or air plasma-induced bonding processes. A portable corona-effect device (Blackhole Laboratory) is also available for small scale or rapid bonding.

(vi) A 3D printer based on the polyjet technology (Objet 30 Pro, Stratasys Ltd). Two proprietary printing materials are currently available: VeroWhite and VeroClear. The VeroWhite material can be deposited with $32 \mu \mathrm{m}$-thick layers, while the VeroClear material is translucent and can be deposited with $16 \mu \mathrm{m}$-thick layers for twice the printing time. The smallest features performed by the machine in the $x y$ coplanar directions are $\sim 60 \pm 10 \mu \mathrm{m}$. When working on microfluidic applications, one of the main disadvantages of such a device is the use of a support material that is printed within the channels and therefore needs to be removed by mechanical and/or chemical means after the printing process. Despite this drawback, the smallest channels produced for our applications had an inner diameter of 300-500 $\mu \mathrm{m}$ (depending on the channel length), while self-supported membranes $\sim 100 \mu \mathrm{m}$ thick were successfully fabricated.

To inspect the various features of the produced devices, quality-control equipment is available for analyses during and after the microfabrication processes. These include the following.

(i) A contact-free profilometer (WLI GmbH) based on white-light interferometry is typically used to map the $3 \mathrm{D}$ topology of micrometre-sized objects in less than a minute with a vertical resolution of $\sim 20 \mathrm{~nm}$. In practice, this equip- 
ment helps in observing the pattern's quality in the resin after the lithography process.

(ii) A custom-made microscope with infinity-corrected optics and a working distance of $34 \mathrm{~mm}$ adapting $5 \times, 10 \times$ and $50 \times$ objectives (Mitutoyo). It is equipped with a Basler camera and allows observing microfluidic systems under circulation with a high resolution.

(iii) A Leica DMS 1000 macroscope with front and back illumination directly casts images on a computer screen for easy manipulation of microfluidic chips.

(iv) Pressure controllers (Elveflow, Fluigent), peristaltic pumps (Ismatec) and syringe pumps (New Era Pump Systems Inc.).

\section{First results}

3.1. A vacuum-compatible microfluidic chip for the in situ study of iron oxide nanoparticles formation using $\mu$-XRF and $\mu$-XAS

In natural water, iron oxyhydroxides are major vectors controlling the behavior of inorganic pollutants such as metals and metalloids. They are indeed known to be strong sorbents of metals and metalloids regarding their high density of binding sites. Under environmental conditions, their structure and their subsequent reactivity is strongly influenced by the physico-chemical conditions that occur during their formation (such as $\mathrm{pH}$, ionic strength, concentration, or the presence of competing cations or anions). As of now, little is known on the species that are formed at the early stage of the reaction and about their evolution with the running time reaction, as a function of physico-chemical conditions. However, the early formed $\mathrm{Fe}$ species are critical regarding the structural diversity of the Fe phases observable in natural systems. To better understand these structures, we followed the nucleation process of $\mathrm{Fe}$ oxides using in situ microfluidic synthesis, which allows the transposing of the time scale into a spatial one (Chan et al., 2007). The evolution of the Fe speciation was followed by Fe $K$-edge X-ray absorption spectroscopy (XAS) during in situ synthesis of $\mathrm{Fe}(\mathrm{III})$ oxides by the oxidation hydrolysis of a $\mathrm{Fe}(\mathrm{II})$ solution, increasing its $\mathrm{pH}$, on the LUCIA beamline (Vantelon et al., 2016).

We have designed a microfluidic device (see Figs. 2 and 3) that is compatible with the primary vacuum $\left(10^{-2} \mathrm{mbar}\right)$

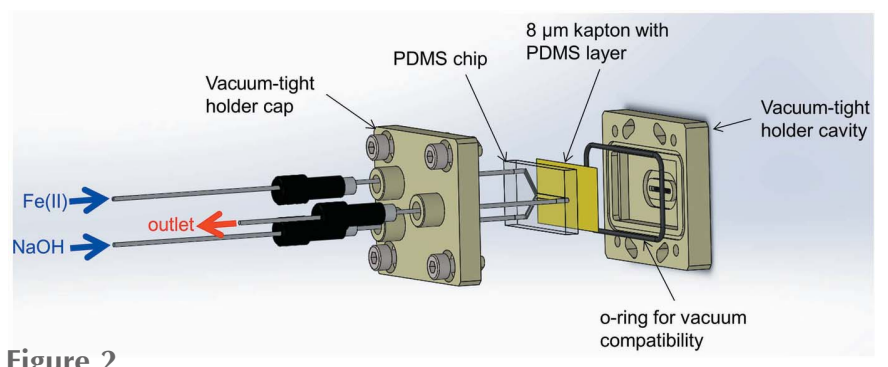

Figure 2

Schematic representation of the vacuum-compatible microfluidic device used on the LUCIA beamline.
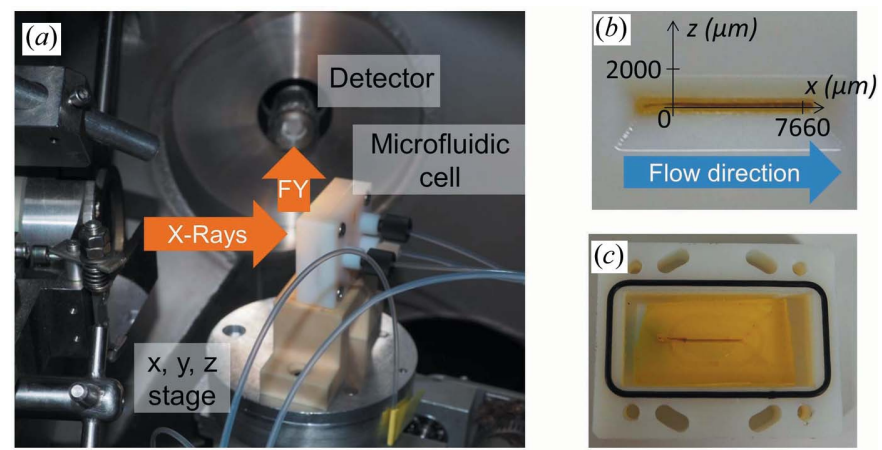

Figure 3

(a) Picture of the experimental setup inside the LUCIA beamline experimental chamber. FY stands for fluorescence yield. (b) Back view of the chip holder during a microfluidic synthesis experiment. The brown color observed inside the channel shows the formation of Fe(III) oxides. (c) Inside view of the vacuum-compatible cell with the PDMS chip at the end of the experiment.

environment of the LUCIA beamline and allows the collection of $\mu$-XAS spectra under microfluidic circulation.

The device consists of a polydimethylsiloxane (PDMS) chip inserted into a vacuum-tight holder. The chip circuit is a $\mathrm{Y}$ shape, with two inlets to introduce the reactants and an observation channel connected to the outlet (see Fig. S1 in the supporting information). The channel was $300 \mu \mathrm{m}$ wide, $500 \mu \mathrm{m}$ deep and $1 \mathrm{~mm}$ long. The chip was microfabricated using an SU-8 mold, which was replicated into a $1 \mathrm{~cm}$-thick PDMS block. The chip was sealed with a $8 \mu \mathrm{m}$-thick Kapton foil on which a $30 \mu \mathrm{m}$ layer of PDMS was spin-coated and activated by plasma treatment. The chip was then inserted into the vacuum-tight holder. The holder consists of a 3D-printed block with a cavity to insert the chip and a cap to close the cavity. The cavity has a $1 \mathrm{~mm}$-wide opening at its back, located in front of the observation channel. The cap has three threaded inlets that allow the connection of tubings to and from the measurement chamber and the chip. The inlet and outlet tubings were connected to the outside of the chamber using feed-through flanges. The holder is made vacuum compatible with an O-ring between the cap and the holder, and by gluing the Kapton foil at the bottom of the holder.

The device was installed on the LUCIA beamline (Vantelon et al., 2016) to follow the evolution of Fe phases during $\mathrm{Fe}(\mathrm{III})$ oxides formation by Fe $K$-edge XAS. The monochromator used was $\mathrm{Si}(111)$ crystals. The energy was calibrated by setting the first inflection point of the $K$-edge of an Fe foil to $7112 \mathrm{eV}$. The beam size was set to $3.5 \mu \mathrm{m} \times 3.5 \mu \mathrm{m}$ for the measurements. Data were collected in fluorescence mode with a monoelement silicon drift detector (SDD) $\left(60 \mathrm{~mm}^{2}\right.$ Brucker). An iron(II) chloride $\left(\mathrm{FeCl}_{2} 0.4 \mathrm{H}_{2} \mathrm{O}\right)$ solution of $1.1 \times$ $10^{-3} \mathrm{~mol} \mathrm{l}^{-1}$ and a sodium hydroxide solution of $1.0 \times$ $10^{-3} \mathrm{~mol} \mathrm{l}^{-1}(\mathrm{NaOH})$ were filled in the inlet channels using syringe pumps (NE-1002X from Pump System Inc.) with a rate of $100 \mu \mathrm{min}^{-1}$. An X-ray fluorescence map of the channel was recorded around the opening with an excitation energy of $7300 \mathrm{eV}$, a step size of $5 \mu \mathrm{m}$ and a counting time of $300 \mathrm{~ms}$ per point. The map is presented in Fig. 4(a) and one can clearly observe three regions: (i) a region without any iron (in green, 


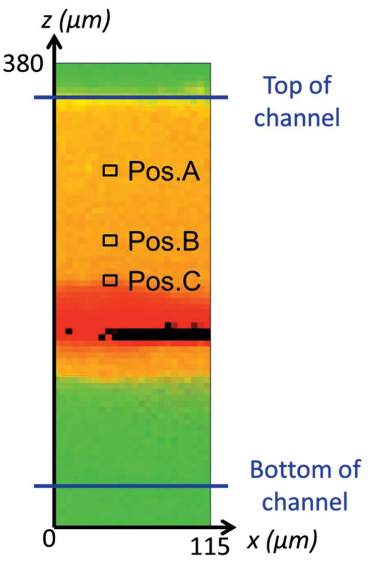

(a) (b)

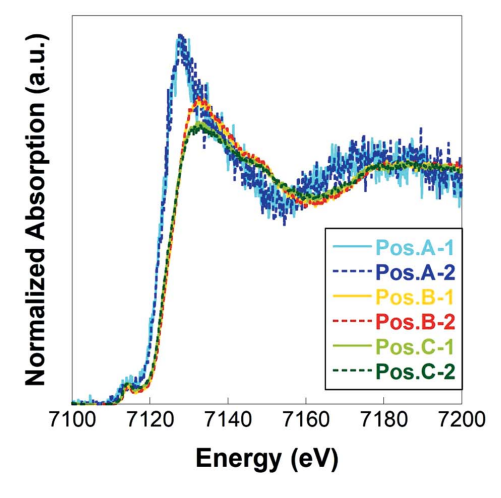

Figure 4

(a) $\mu$-XRF map recorded on the observation channel. Green stands for the silicon of the chip, orange to red stands for the iron (low and high concentration, respectively) and black indicates a concentration of iron so high that the detector was saturated. (b) Normalized XANES spectra at the $\mathrm{Fe} K$-edge recorded on the positions indicated on the $\mu$-XRF map. The positions where the spectra were collected are reported in $(a)$ as positions A, B and C. The collection of spectra was duplicated; the two successive XANES spectra being labeled 1 and 2, respectively.

at the bottom of the channel), which is the region where the $\mathrm{NaOH}$ solution flows; (ii) a region with low iron content (in orange, at the top of the channel), which is the region where the injected $\mathrm{FeCl}_{2}$ flows; and (iii) an iron-enriched region (in red, in the center of the channel), where the interaction between the $\mathrm{FeCl}_{2}$ and $\mathrm{NaOH}$ solutions occurs and $\mathrm{Fe}$ (III) oxide nanoparticles precipitate. X-ray absorption near-edge structure (XANES) spectra were collected at the Fe $K$-edge at different positions in the channel, going from the $\mathrm{Fe}(\mathrm{II})$ solution to the $\mathrm{Fe}$ (III) precipitates (from the top to the center of the channel). Data are reported in Fig. 4(b). The edge energy shift between the first spectrum and the others indicates that the starting $\mathrm{Fe}$ (II) ions have been oxidized at the contact with $\mathrm{NaOH}$ to form $\mathrm{Fe}$ (III) oxide nanoparticles. The different shapes observed from position B and position $\mathrm{C}$ spectra are caused by the increasing non-corrected dead-time subsequent to the accumulation of $\mathrm{Fe}$ (III) in the channel. The collection of two consecutive $\mu$-XAS spectra on each spot [referred to as spectra 1 and 2 in Fig. 4(b)] shows that there are no beaminduced damages and deposition on the window. The further study of the iron oxides nucleation growth will be to create more complex systems using the addition of cations, ions and organic matter to make it closer to environmental conditions. However, these

Figure 5 preliminary results demonstrate that the collection of $\mu$-XRF (micro X-ray fluorescence) and $\mu$-XANES spectra in microfluidic devices are possible under vacuum, which opens up a wide range of studies in the tender and soft X-ray energy ranges for the study of light elements.

\subsection{Chemical kinetics of nanoparticle dilution using a 3D-printed and PDMS microfluidic mixer}

The dynamics of protein (un)folding nanoparticles formation or fiber assembly can be studied efficiently using synchrotron-based X-ray scattering or spectroscopic techniques. In situ flow-cell devices are required in order to follow such fast kinetic phenomena. Although stop-flow systems have been traditionally used for these applications, the advent of 3D-printing systems at reasonable costs and with good resolution makes it a very attractive alternative (Au et al., 2016). Although they require more advanced equipment and knowhow, microfluidic chips can also be used as versatile and reactive mixing and/or flowing systems suitable for in situ $\mathrm{X}$-ray diffusion techniques.

We describe here a PDMS-based microfluidic chip (see Figs. 5 and S2) for time-resolved in situ small-angle X-ray scattering/wide-angle X-ray scattering (SAXS/WAXS) data collection on liquid solutions. It is made of three parts: the upper layer containing channels of $180 \mu \mathrm{m} \times 180 \mu \mathrm{m}$ (height $\times$ width), the lower part consisting of a spin-coated layer of PDMS $(100 \mu \mathrm{m})$ on top of a Kapton foil $(25 \mu \mathrm{m})$, and a 3Dprinted backbone for stiffness and to prevent PDMS from leaking onto the area of measurement during the fabrication process. Thanks to this design, the X-ray beam only needs to go through two thin layers of PDMS (both $100 \mu \mathrm{m}$ thick) and a
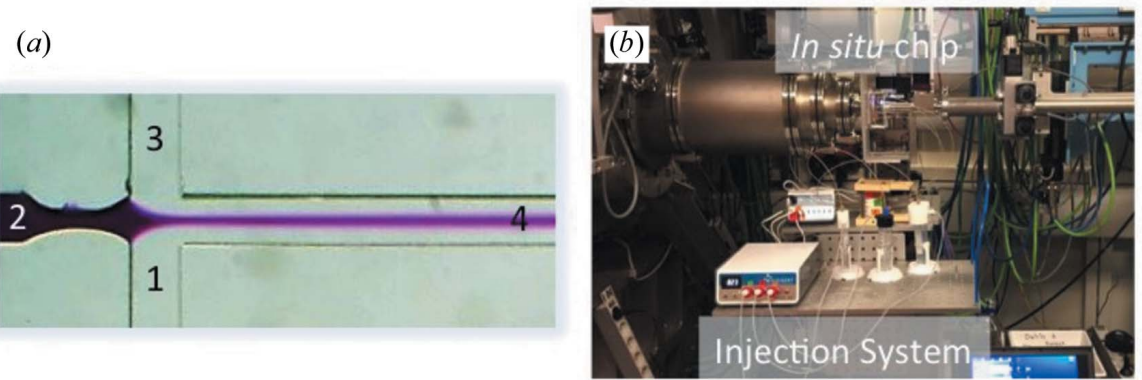

(c)
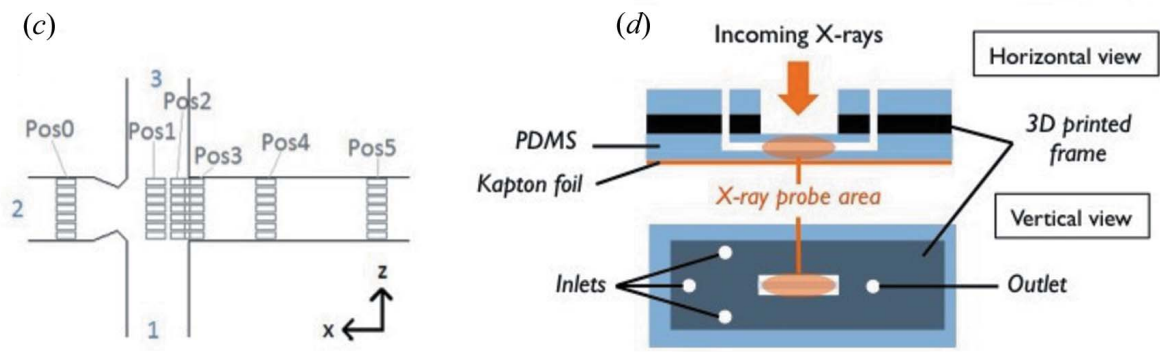

(a) Picture of the PDMS chip showing all the channels and the flow-focusing effect, $(b)$ picture of the in situ cell and setup installed on the SWING beamline, $(c)$ sketch of the positions where SAXS data were recorded into the PDMS chip and $(d)$ scheme of the microfluidic chip design. Further information on the fabrication process can be found in Fig. S2. 
Kapton foil ( $25 \mu \mathrm{m}$ thick), thus resulting in minimal parasitic scattering.

The PDMS chip was designed as a four-channels cross with a restriction in the second channel [see Fig. 5(a)]. This geometry allows using the flow-focusing technique in order to investigate the first few milliseconds of assembly or reaction processes (Knight et al., 1998; Otten et al., 2005). The principle of flow focusing consists of squeezing a central stream of liquid (liquid in channel 2) arriving at the four channels intersection by the two side streams coming from channels 1 and 3 [see Fig. 5(a)]. As a result, a thin-sheathed stream is produced in the channel 4 outlet. This flowing configuration is a faster alternative to turbulent mixing since, at such small scales, solutes coming from channels 1 and 3 rapidly diffuse across the stream from channel 2 (Knight et al., 1998).

We have used the PDMS chip to record SAXS data on $\mathrm{Au}$ nanoparticles (see Section S2 in the supporting information for Au nanoparticles preparation). SAXS data were recorded at $12 \mathrm{keV}$ on the SWING (David \& Pérez, 2009) beamline of SOLEIL and the $q$ range was chosen in order to cover the full form-factor range of 5 and $10 \mathrm{~nm}$-diameter $\mathrm{Au}$ nanoparticles spheres, corresponding to a distance of $1 \mathrm{~m}$ between the sample and the detector. The chip was placed into the beam path and connected to a piezo-controlled pressure system (Fluigent) for flow control and regulation [Fig. 5(b)]. SAXS images were recorded at the X-ray probe area of the PDMS chip [Fig. $5(d)$ ].

First, SAXS images were recorded with citrate buffer flowing through the channels in order to subtract background from the sample measurements. Then, using the pressurecontrol system, two batches of $10 \mathrm{n} M$ Au nanoparticles solutions (5 and $10 \mathrm{~nm}$ in diameter) were injected with a steady flow rate: $5 \mu \mathrm{min}^{-1}$ in channel 2 of the PDMS chip. The citrate buffer was injected at $11 \mu \mathrm{min}^{-1}$ in channels 1 and 3 . SAXS images were recorded at the same position in the chip for the buffer and for the samples thanks to an accurate motorized table, 2D images were reduced to $1 \mathrm{D}$ curves and background was subtracted using the Foxtrot software (SOLEIL custom-made software). Despite the small volume probed by the X-ray beam (channel height is $180 \mu \mathrm{m}$ ) and the low-intensity signal obtained, curves were fitted using the Irina package (Ilavsky \& Jemian, 2009), giving a radius for $\mathrm{Au}$ nanoparticles of $29.87 \pm 0.06 \AA$ (Fig. S4). The measured radius is therefore in good agreement with the one announced by the manufacturer $(25 \AA)$. As the signal was very low, we decided to use $10 \mathrm{~nm}$-diameter Au nanoparticles in order to study their dilution in the chip.

We first recorded SAXS images at position 0 at different vertical spots. The five resulting curves show that there is no decrease in intensity at small $q$ [Fig. 6(a) red circle] and that there is no modification of the nanoparticles form factor, while vertically scanning the channel. This is expected but confirms the reproducibility of the measurement on different vertical spots. Fitting those curves resulted in $\mathrm{Au}$ nanoparticles of $45.55 \pm 0.07 \AA$ in radius [Fig. 6(c)]. SAXS data were then collected at positions $1-5$ at several vertical spots, corresponding to different positions in the buffer-nanoparticle solution gradient at different times of the diffusion process. While moving towards position 5, we observe as expected a dilution of the nanoparticles solution along channel 4 . The dilution is visible on SAXS curves as the intensity at small $q$ is increasing from the channel border towards the channel center [Fig. 6(b), red oval]. The signal being still strong, we were able to fit these curves. A radius of $46.71 \pm 0.09 \AA$ was found [Fig. $6(d)$ ], in good agreement with the announced radius $(50 \AA)$. It should be noted that subtracting the chips/ buffer background signal from the sample signal is mandatory, which requires foreseeing the position where the sample will be measured. Despite this procedure, small differences in the background can still be observed between the signal from the chip loaded with buffer and from the chip loaded with buffer and sample [Fig. 6(a), green curve]. This can be explained by slight changes in the optical path length because of the higher pressure required to flow the sample into the chip.

A fully 3D-printed in situ liquid flow cell was also fabricated and used to collect SAXS data. It is made of a single block VeroClear piece, as shown in Fig. S3. The only post-printing processing consisted of removing the printing support material from the outside of the block and the inside of the channel (using a thin needle), and machining the threads for the inlet and outlet liquid connectors. The channel inner diameter was $500 \mu \mathrm{m}$ and the front and back membranes were $\sim 100 \mu \mathrm{m}$ thick. PTFE (polytetrafluoroethylene) tubings were

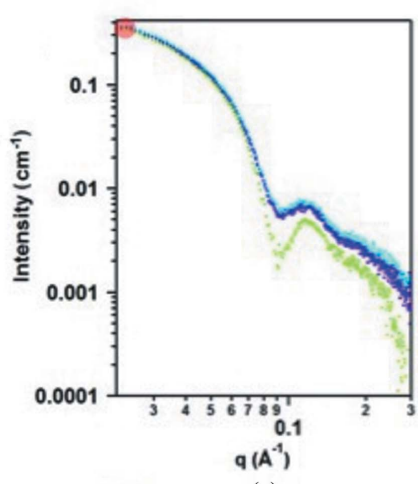

(a)

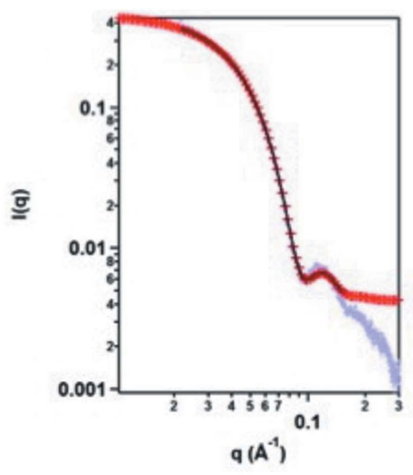

(c)

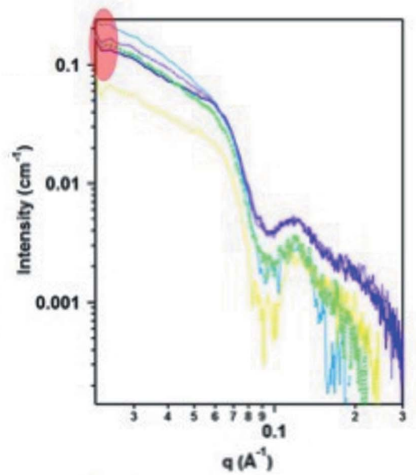

(b)

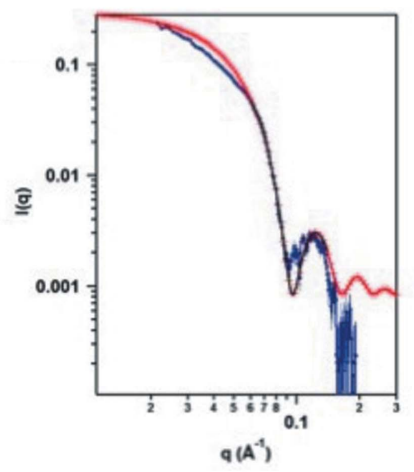

(d)
Figure 6

SAXS curves of $10 \mathrm{~nm}$-diameter Au nanoparticles in the PDMS chips at different heights for position $0(a)$ and position $5(b)$. Fitted curves (black) of $10 \mathrm{~nm}$-diameter $\mathrm{Au}$ nanoparticles recorded in the center of channel 4 at positions $0(c)$ and $5(d)$. Theoretical curves are shown in red. 
connected to the cell's inlet and outlet, and a $10 \mathrm{n} M$ solution of gold nanoparticle ( $5 \mathrm{~nm}$ in diameter) was flown through the cell. Although there was no mixing possible in this flow cell, the quality of the signal was much higher because of the larger volume probed by the X-rays (the channel is $500 \mu \mathrm{m}$ thick versus $180 \mu \mathrm{m}$ for the PDMS chip). The data collected with this cell and the associated curve fittings can be found in Fig. S4.

Thanks to 3D printing and to soft-lithography techniques, we have designed and manufactured chips in a very short time (1-2 d), in which we can record form factors of 5 and $10 \mathrm{~nm}$ diameter Au nanoparticles at a concentration of $10 \mathrm{n} M$ for two different optical paths $(500$ and $180 \mu \mathrm{m})$. Moreover, in the case of the PDMS chip, we have shown that the flow-focusing technique can be applied to record form factors of nanoparticles while they are being diluted and to follow this process in situ. These experimental setups could be easily used as disposable 3D-printed flow cells in order to replace quartz capillaries for inert samples and, for the flow-focusing device, as a convenient means of probing fast kinetic reactions or assembly processes.

\subsection{Macromolecular serial crystallography using microfluidic-based trapping chips}

The field of structural biology consists of a plethora of complementary methods aimed at obtaining functional information on complex macromolecular objects. Among the most popular techniques, macromolecular crystallography (MX) has been greatly recognized in obtaining near-atomic resolution details on such molecules. A typical MX experiment implies the use of cryo-protected protein crystals, the data measurements being performed at cryogenic temperatures in order to limit the propagation of radiation damage generated by the strong X-rays hitting the biological crystals. Although the use of cryogenic conditions and protocols appears as mandatory in accepted MX diffraction experiments, it shows some deleterious effects on the crystals' integrity and, for some specific systems, prevents dynamical studies. In such a context and without proper experimental methods available, molecular dynamics simulations appear to be an elegant approach in tackling these restricted applications (Vasil'ev \& Bruce, 2006). The latest developments of X-ray free-electron laser (XFEL) sources moved the field to a new horizon where crystals not cryo-protected are measured once before being destroyed by the highly intense beam of the XFEL short pulses, yet open to diffraction measurements free of noticeable radiation damage deleterious to the understanding of biological processes (Chapman et al., 2011). This new technique of serial femtosecond crystallography (SFX) has rapidly evolved and produced spectacular results linked to better understanding complicated enzymatic machineries (MartinGarcia et al., 2016; Kern et al., 2018). Nevertheless, the SFX approach remains extremely challenging in terms of sample injection into the X-ray interaction point, which eventually reflects on the capacity to produce crystalline samples and produce with sufficiently good quality.
Alternative strategies to deliver crystals to an X-ray beam are being investigated and notably include microfluidic devices. Several such systems have recently been proposed for in situ protein crystallography, where crystals can be studied directly in their growing conditions without any further transfer or freezing process (Pinker et al., 2013). Other systems immobilize the objects to be studied by circulating them within their growing media in a microfluidic environment with an array of traps along the path of the chip (Lyubimov et al., 2015). However, most of these systems remain proof-of-principle demonstrations and were not fully applied to address specific biological questions.

Inspired by these previous studies, we developed and used several systems to quickly handle macromolecular crystals. Three-dimensional printed-capillary holders were designed for adapting diffraction experiments to in situ capillary-grown crystals (Fig. 7). Using the 3D printer of the MF-Lab, a rigid frame was fabricated on which a magnetic pin, an injection tube and the capillary to receive the crystals were assembled. Crystals grown in another microfluidic system (Gerard et al., 2017) were injected from the storage capillary and into the $\mathrm{X}$-ray diffraction capillary using a pressure controller and syringe pumps while connecting both capillaries with standard high-performance liquid chromatography tubing tools. After detaching the storage capillary, the frame was mounted on a goniometer of the PROXIMA-1 beamline (Coati et al., 2017), as depicted in Fig. 7(b), and diffraction data were collected. Although in the current setup both storage and X-ray

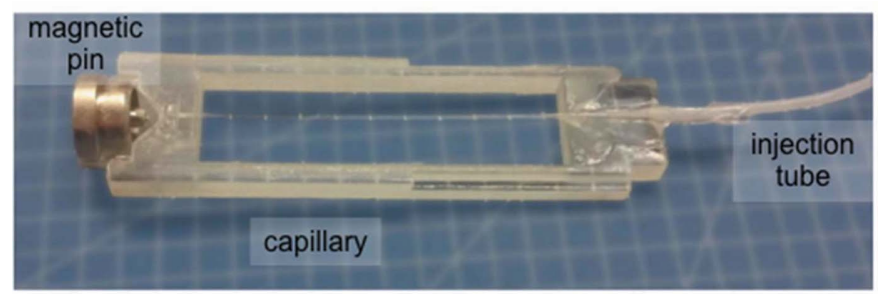

(a)

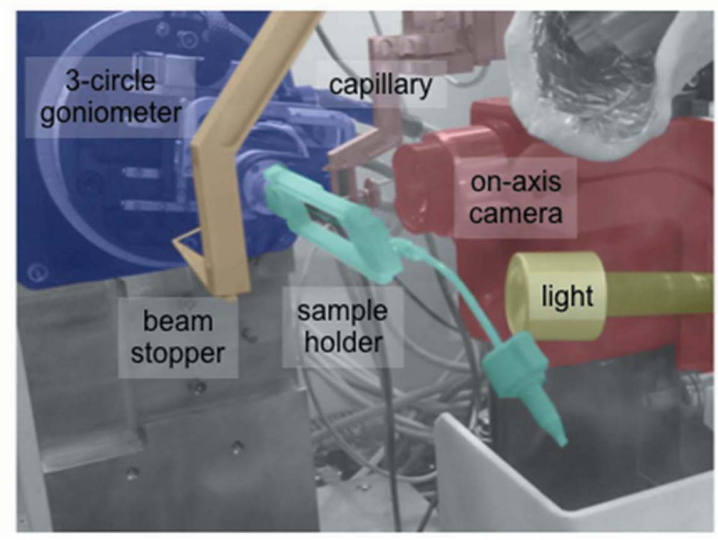

(b)

Figure 7

(a) Capillary and injection tube mounted on a magnetic pin for X-ray diffraction experiments. (b) 3D-printed adapting tool (cyan) mounted on a three-circle goniometer (purple) at the PROXIMA-1 beamline. The tubing used to load the samples remains visible. 

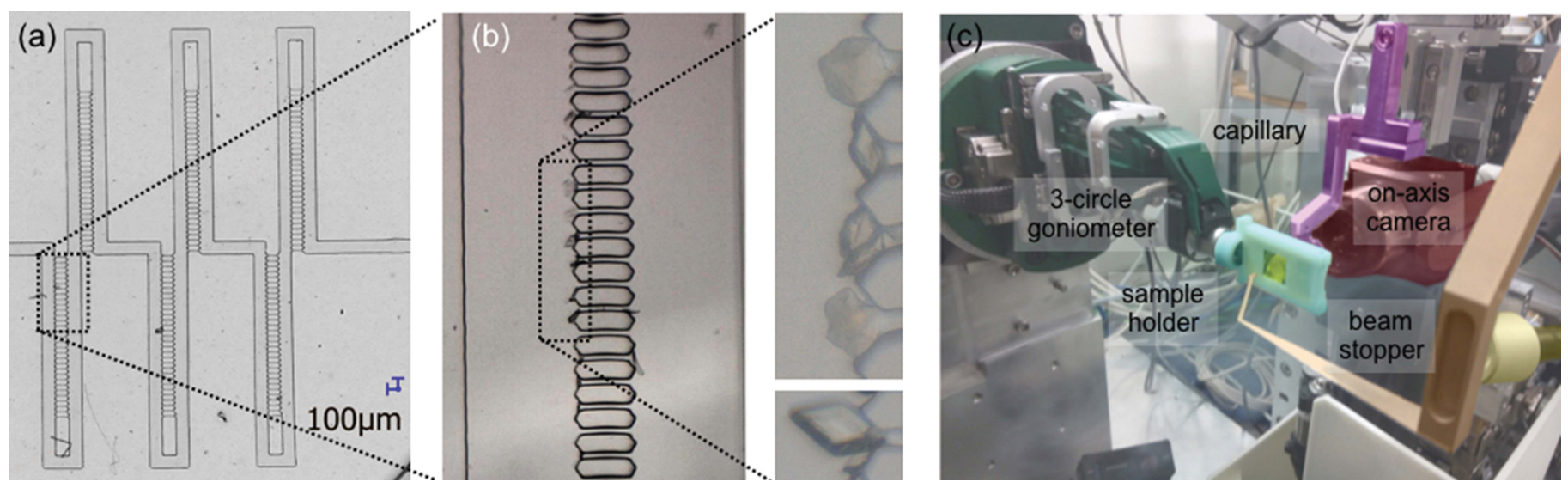

Figure 8

(a) Pattern overview inside the PDMS/Kapton chip used for trapping biological objects. A total of 180 traps arranged in three 'way and back' lines are shown in this view. (b) Enlarged view of trapped crystals within the chip. (c) 3D-printed chip holder (cyan) mounted on a three-circle goniometer (green).

diffraction capillaries were disconnected before the X-ray experiments, one could foresee some fields of interest in collecting X-ray diffraction data on solutions constantly flowing through the capillary, notably when screening for crystal conditions in an in situ crystal-growth approach.

A PDMS-based microfluidic chip was also designed and fabricated (see Fig. S5) in which crystals were loaded and trapped at known positions ready for X-ray diffraction experiments [Figs. $8(a)$ and $8(b)$ ]. For X-ray diffraction data recording, this chip was inserted into a custom-designed 3Dprinted chip holder, which includes a magnetic pin. The chip was loaded with protein crystals $(40 \mu \mathrm{m}$ in the longest dimension) by circulating a suspension of crystals in their mother liquor using a syringe pump. The chip holder was mounted on a three-circle goniometer at PROXIMA-1 and serial-crystallography experiments were recorded at an energy of $12.67 \mathrm{keV}$ on a Pilatus 6M (Dectris Ltd) detector [Fig. 8(c)]. To obtain a complete data set, data collection from three isolated crystals exposed over an oscillation angle of $50^{\circ}$ each were merged with good statistics up to a resolution of $1.6 \AA$ (Table S1). The size of the X-ray beam at the sample position was restricted to $40 \mu \mathrm{m} \times 20 \mu \mathrm{m}$ for a photon flux of $1 \times 10^{10}$ photons s $\mathrm{sm}^{-1}$. Inspection of the diffraction images for each of the three selected data sets did not show noticeable radiation damage.

\section{Conclusion}

The availability of a dedicated microfluidic laboratory within a light-producing facility is ideal for on-demand and rapid fabrication of microfluidic systems that combine both the requirements of sample delivery and photon-based techniques. The variety of instruments available for both chip fabrication and analysis allows a fast response to the user's demands for off- and on-line requests. Maskless lithography and 3D-printing possibilities are instrumental in providing the emergency response that is required by typical synchrotron workflow. Beyond user-driven considerations, the presence of an in-house microfluidic laboratory at a synchrotron promotes the development of novel interaction points between spectroscopic and structural techniques and sample-delivery systems. We should stress once more that the high photon density of modern synchrotron sources can pose a serious threat to the sample's integrity, and microfluidic systems can provide solutions to this issue by rapidly regenerating the sample or removing the excess of heat or radicals generated by the beam.

In terms of scientific applications, microfluidic systems will be very helpful in providing liquid environments to solid samples such as crystals of biological objects, nanoparticles or micrometre-sized materials as they are analyzed by a photon beam. Drug discovery should be facilitated by the possibility to change the environment of a protein crystal at room temperature and in situ, the structure of reaction intermediates in the synthesis of nanoparticles can also be tracked in situ and the solid-liquid interface of catalytic films can be observed under operating conditions.

Although the microfluidic laboratory at SOLEIL is not opened yet to external users via the regular proposal review committees, it is available to all of the SOLEIL staff, associates and collaborators. Users interested in this facility should contact the laboratory manager or any beamline scientist from SOLEIL with whom a collaboration centered on microfluidics is to be developed.

\section{Acknowledgements}

Dr Paul Dumas, Dr Gabriel David and Ms. Valérie Rouam are greatly acknowledged for their help in setting up the microfluidic laboratory and its equipment. IC performed the experiments under a collaborative research program between SOLEIL and the SERVIER Research Institute. The LUCIA, SWING and PROXIMA-1 teams are greatly acknowledged for their assistance in data collection during commissioning beam time. 


\section{Funding information}

LMGC acknowledges funding from the European Union's Horizon 2020 research and innovation programme under Grant Agreement No. 708130.

\section{References}

Au, A. K., Huynh, W., Horowitz, L. F. \& Folch, A. (2016). Angew. Chem. Int. Ed. 55, 3862-3881.

Chan, E. M., Marcus, M. A., Fakra, S., ElNaggar, M., Mathies, R. A. \& Alivisatos, A. P. (2007). J. Phys. Chem. A, 111, 12210-12215.

Chapman, H. N., Fromme, P., Barty, A., White, T. A., Kirian, R. A., Aquila, A., Hunter, M. S., Schulz, J., DePonte, D. P., Weierstall, U., Doak, R. B., Maia, F. R. N. C., Martin, A. V., Schlichting, I., Lomb, L., Coppola, N., Shoeman, R. L., Epp, S. W., Hartmann, R., Rolles, D., Rudenko, A., Foucar, L., Kimmel, N., Weidenspointner, G., Holl, P., Liang, M., Barthelmess, M., Caleman, C., Boutet, S., Bogan, M. J., Krzywinski, J., Bostedt, C., Bajt, S., Gumprecht, L., Rudek, B., Erk, B., Schmidt, C., Hömke, A., Reich, C., Pietschner, D., Strüder, L., Hauser, G., Gorke, H., Ullrich, J., Herrmann, S., Schaller, G., Schopper, F., Soltau, H., Kühnel, K., Messerschmidt, M., Bozek, J. D., Hau-Riege, S. P., Frank, M., Hampton, C. Y., Sierra, R. G., Starodub, D., Williams, G. J., Hajdu, J., Timneanu, N., Seibert, M. M., Andreasson, J., Rocker, A., Jönsson, O., Svenda, M., Stern, S., Nass, K., Andritschke, R., Schröter, C., Krasniqi, F., Bott, M., Schmidt, K. E., Wang, X., Grotjohann, I., Holton, J. M., Barends, T. R. M., Neutze, R., Marchesini, S., Fromme, R., Schorb, S., Rupp, D., Adolph, M., Gorkhover, T., Andersson, I., Hirsemann, H., Potdevin, G., Graafsma, H., Nilsson, B. \& Spence, J. C. H. (2011). Nature, 470, 73-77.

Coati, A., Chavas, L. M. G., Fontaine, P., Foos, N., Guimaraes, B., Gourhant, P., Legrand, P., Itie, J.-P., Fertey, P., Shepard, W., Isabet, T., Sirigu, S., Solari, P.-L., Thiaudiere, D. \& Thompson, A. (2017). Eur. Phys. J. Plus, 132, 174.

David, G. \& Pérez, J. (2009). J. Appl. Cryst. 42, 892-900.

Gerard, C. J. J., Ferry, G., Vuillard, L. M., Boutin, J. A., Chavas, L. M. G., Huet, T., Ferte, N., Grossier, R., Candoni, N. \& Veesler, S. (2017). Acta Cryst. F73, 574-578.

Hemmerle, A., Fragneto, G., Daillant, J. \& Charitat, T. (2016). Phys. Rev. Lett. 116, 228101.

Ilavsky, J. \& Jemian, P. R. (2009). J. Appl. Cryst. 42, 347-353.
Joanicot, M. \& Ajdari, A. (2005). Science, 309, 887-888.

Kern, J., Chatterjee, R., Young, I. D., Fuller, F. D., Lassalle, L., Ibrahim, M., Gul, S., Fransson, T., Brewster, A. S., Alonso-Mori, R., Hussein, R., Zhang, M., Douthit, L., de Lichtenberg, C., Cheah, M. H., Shevela, D., Wersig, J., Seuffert, I., Sokaras, D., Pastor, E., Weninger, C., Kroll, T., Sierra, R. G., Aller, P., Butryn, A., Orville, A. M., Liang, M., Batyuk, A., Koglin, J. E., Carbajo, S., Boutet, S., Moriarty, N. W., Holton, J. M., Dobbek, H., Adams, P. D., Bergmann, U., Sauter, N. K., Zouni, A., Messinger, J., Yano, J. \& Yachandra, V. K. (2018). Nature, 563, 421-425.

Knight, J. B., Vishwanath, A., Brody, J. P. \& Austin, R. H. (1998). Phys. Rev. Lett. 80, 3863-3866.

Lyubimov, A. Y., Murray, T. D., Koehl, A., Araci, I. E., Uervirojnangkoorn, M., Zeldin, O. B., Cohen, A. E., Soltis, S. M., Baxter, E. L., Brewster, A. S., Sauter, N. K., Brunger, A. T. \& Berger, J. M. (2015). Acta Cryst. D71, 928-940.

Martin-Garcia, J. M., Conrad, C. E., Coe, J., Roy-Chowdhury, S. \& Fromme, P. (2016). Arch. Biochem. Biophys. 602, 32-47.

Otten, A., Köster, S., Struth, B., Snigirev, A. \& Pfohl, T. (2005). J. Synchrotron Rad. 12, 745-750.

Pinker, F., Brun, M., Morin, P., Deman, A.-L., Chateaux, J.-F., OliéRic, V., Stirnimann, C., Lorber, B., Terrier, N., Ferrigno, R. \& Sauter, C. (2013). Cryst. Growth Des. 13, 3333-3340.

Polte, J., Erler, R., Thünemann, A. F., Sokolov, S., Ahner, T. T., Rademann, K., Emmerling, F. \& Kraehnert, R. (2010). ACS Nano, 4, 1076-1082.

Qin, D., Xia, Y. \& Whitesides, G. M. (2010). Nat. Protoc. 5, 491-502.

Reich, C., Hochrein, M. B., Krause, B. \& Nickel, B. (2005). Rev. Sci. Instrum. 76, 095103.

Song, H. \& Ismagilov, R. F. (2003). J. Am. Chem. Soc. 125, 14613-14619.

Tabeling, P. (2005). Introduction to Microfluidics. Oxford University Press.

Vantelon, D., Trcera, N., Roy, D., Moreno, T., Mailly, D., Guilet, S., Metchalkov, E., Delmotte, F., Lassalle, B., Lagarde, P. \& Flank, A.-M. (2016). J. Synchrotron Rad. 23, 635-640.

Vasil'ev, S. \& Bruce, D. (2006). Biophys. J. 90, 3062-3073.

Whitesides, G. M. (2006). Nature, 442, 368-373.

Zarzar, L. D., Sresht, V., Sletten, E. M., Kalow, J. A., Blankschtein, D. \& Swager, T. M. (2015). Nature, 518, 520-524.

Zheng, B., Tice, J. D., Roach, L. S. \& Ismagilov, R. F. (2004). Angew. Chem. Int. Ed. 43, 2508-2511. 